\title{
COALESCENCE IN SUBCRITICAL BELLMAN-HARRIS AGE-DEPENDENT BRANCHING PROCESSES
}

\author{
JYY-I HONG, ${ }^{*}$ Waldorf College
}

\begin{abstract}
We consider a continuous-time, single-type, age-dependent Bellman-Harris branching process. We investigate the limit distribution of the point process $A(t)=\left\{a_{t, i}: 1 \leq\right.$ $i \leq Z(t)\}$, where $a_{t, i}$ is the age of the $i$ th individual alive at time $t, 1 \leq i \leq Z(t)$, and $Z(t)$ is the population size of individuals alive at time $t$. Also, if $Z(t) \geq k, k \geq 2$, is a positive integer, we pick $k$ individuals from those who are alive at time $t$ by simple random sampling without replacement and trace their lines of descent backward in time until they meet for the first time. Let $D_{k}(t)$ be the coalescence time (the death time of the last common ancestor) of these $k$ random chosen individuals. We study the distribution of $D_{k}(t)$ and its limit distribution as $t \rightarrow \infty$.
\end{abstract}

Keywords: Branching process; coalescence; subcritical; Bellman; Harris; age dependent; line of descent

2010 Mathematics Subject Classification: Primary 60J80

Secondary 60G50

\section{Introduction}

\subsection{Definition of Bellman-Harris branching processes}

We consider a single-type, continuous-time, age-dependent branching process such that each individual lives for a random amount of time, say $L$, with distribution function $G$ and, upon death, produces a random number $\xi$ of children according to the offspring distribution $\left\{p_{j}\right\}_{j \geq 0}$ with $L$ and $\xi$ independent. All individuals live and produce children independently of each other and with the same distributions. (See [3, Chapter 4].)

Let $Z(t)$ be the population at time $t$, i.e. the number of individuals alive at time $t$. Then $\{Z(t): t \geq 0\}$ is called a continuous-time, single-type, age-dependent Bellman-Harris branching process with lifetime distribution $G(\cdot)$ and offspring distribution $\left\{p_{j}\right\}_{j \geq 0}$.

Let $m \equiv \sum_{j=1}^{\infty} j p_{j}$. The Bellman-Harris branching process is called a supercritical, critical, or subcritical process according to whether $1<m<\infty, m=1$, or $m<1$.

For the lifetime distribution $G$, we assume throughout that $G(0+)=0$. This together with finite mean (i.e. $m<\infty$ ) guarantees the almost-sure finiteness of the process for all time $t>0$, i.e. $\mathbb{P}(Z(t)<\infty)=1$ for all $0<t<\infty$.

Definition 1.1. The Malthusian parameter for $m$ and $G$ is the root $\alpha$ in $\mathbb{R}$ (provided it exists) such that

$$
m \int_{0}^{\infty} \mathrm{e}^{-\alpha x} \mathrm{~d} G(x)=1 .
$$

Received 13 July 2012; revision received 12 September 2012.

* Postal address: Department of Mathematics, Waldorf College, 106 South 6th Street, Forest City, Iowa 50436, USA.

Email address: hongjyyi@gmail.com 
Due to the monotonicity of the left-hand side of the above equation as a function of $\alpha$, such a root, when it exists, it is always unique. Also, such a Malthusian parameter $\alpha$ always exists and is necessarily nonnegative when $m \geq 1$.

Let $f$ be the generating function of the offspring distribution, i.e.

$$
f(s)=\sum_{j=0}^{\infty} p_{j} s^{j}, \quad 0 \leq s \leq 1 .
$$

Let

$$
F(s, t) \equiv \sum_{j=0}^{\infty} \mathbb{P}(Z(t)=j \mid Z(0)=1) s^{j}, \quad 0 \leq s \leq 1
$$

Thus,

$$
F(s, t) \equiv \mathbb{E}\left(s^{Z(t)} \mid \text { a newborn ancestor at time } 0\right)=\mathbb{E}\left(s^{Z(t)}: L_{0}>t\right)+\mathbb{E}\left(s^{Z(t)}: L_{0} \leq t\right),
$$

where $L_{0}$ is the lifetime of the ancestor. On the event $\left\{L_{0} \leq t\right\}$,

$$
Z(t)=\sum_{j=1}^{\xi_{0}} Z_{j}\left(t-L_{0}\right)
$$

where $\xi_{0}$ is the number of offspring of the ancestor and $\left\{Z_{j}\left(t-L_{0}\right): t \geq L_{0}\right\}$ is the branching process initiated by the $j$ th offspring of the ancestor, $j=1,2, \ldots, \xi_{0}$. Thus,

$$
F(s, t)=s(1-G(t))+\int_{[0, t]} f(F(s, t-x)) \mathrm{d} G(x) ;
$$

$F(s, t)$ can be shown to be the unique bounded solution of the above integral equation (see [3, pp. 139-140]). Thus, $F$ is fully determined by the pair $(f, G)$.

We now present some well-known results for Bellman-Harris processes. (See [3] for proofs.) Let $q$ be the probability of extinction, i.e.

$$
q \equiv \mathbb{P}(Z(t)=0 \text { for some } 0<t<\infty \mid Z(0)=1) .
$$

It is known that $q=1$ in the critical and subcritical cases $(0<m \leq 1)$.

Theorem 1.1. If $m \neq 1,0<\gamma=f^{\prime}(q), G$ is nonlattice, the Malthusian parameter $\alpha$ for $\gamma$ and $G$ exists, and $\mu_{\alpha}=\gamma \int t \mathrm{e}^{-\alpha t} \mathrm{~d} G(t)<\infty$, then

$$
\lim _{t \rightarrow \infty} \mathrm{e}^{-\alpha t}(q-F(s, t)) \equiv Q(s) \quad \text { exists for } 0 \leq s \leq 1 .
$$

Furthermore,

$$
Q(s) \equiv 0 \Longleftrightarrow m<1 \text { and } \sum_{j=1}^{\infty}(j \log j) p_{j}=\infty .
$$

Theorem 1.2. Let $0<m<1$ and $\sum_{j=1}^{\infty}(j \log j) p_{j}<\infty$. Assume that the Malthusian parameter $\alpha$ for $m$ and the lifetime distribution $G$ exists and that $\int_{0}^{\infty} t \mathrm{e}^{-\alpha t} \mathrm{~d} G(t)<\infty$. Then

(a) for all $j \geq 1$,

$$
\lim _{t \rightarrow \infty} \mathbb{P}(Z(t)=j \mid Z(t)>0)=b_{j}
$$

exists, $\sum_{j=1}^{\infty} b_{j}=1$, and $\sum_{j=1}^{\infty} j b_{j}<\infty$,

(b) $\mathbb{P}(Z(t)>0) \sim c \mathrm{e}^{\alpha t}$ for some $0<c<\infty$. 


\subsection{The age chart}

Since, by Theorem 1.2(a), conditioned on the event of nonextinction, the population $Z(t)$ of a single-type, continuous-time, age-dependent subcritical branching process will converge to a proper real-valued random variable in distribution as $t \rightarrow \infty$, the question of the convergence of the age chart of the individuals alive at time $t$ is of interest. Let $a_{t, i}$ be the age of the $i$ th individual alive at time $t, 1 \leq i \leq Z(t)$. Then, $\left\{A(t) \equiv\left\{a_{t, i}: 1 \leq i \leq Z(t)\right\}, t \geq 0\right\}$ is a point process. In this paper, the limit distribution of $A(t)$ as $t \rightarrow \infty$ conditioned on the event $\{Z(t)>0\}$ will be discussed.

\subsection{The coalescence problem}

Suppose that, for $t>0, Z(t) \geq k$. Now, pick $k$ individuals at random from the population alive at time $t$ by simple random sampling without replacement. Trace their lines of descent backward in time till they meet. Let $D_{k}(t)$ be the coalescence time of these $k$ individuals alive at time $t$. We call this common ancestor who died at time $D_{k}(t)$ their last common ancestor. In this paper, the limit behaviors of the distributions of $D_{k}(t)$ as $t \rightarrow \infty$ for $k \geq 2$ is studied for the subcritical age-dependent Bellman-Harris branching process.

The analog of Theorem 2.2 (below, the result on the coalescence time) for the discrete-time, single-type Galton-Watson branching processes has been addressed in [1] (for supercritical and explosive cases) and [2] (for critical and subcritical cases). Also, Lambert [7] considered the discrete and continuous (time and state space) settings for subcritical cases and Hong dealt with continuous-time, age-dependent supercritical Bellman-Harris branching processes in [5]. For the results on multitype discrete-time processes, see Hong [5] for supercritical, critical, and subcritical cases.

\section{Main results}

The first result we establish for the subcritical case is the convergence of the age chart of the population.

Theorem 2.1. Let $0<m<1$ and $\sum_{j=1}^{\infty}(j \log j) p_{j}<\infty$. Assume that the lifetime distribution $G$ is nonlattice, $G(0+)=0$ and such that the Malthusian parameter $\alpha$ exists, and $\int_{0}^{\infty} t \mathrm{e}^{-\alpha t} \mathrm{~d} G(t)<\infty$. Then the following statements hold.

(a) Conditioned on the event $\{Z(t)>0\}$, the point process

$$
A(t) \equiv\left\{a_{t, i}: 1 \leq i \leq Z(t)\right\}
$$

converges in distribution as $t \rightarrow \infty$ to a point process

$$
\tilde{A} \equiv\left\{\tilde{a}_{i}: 1 \leq i \leq Y\right\},
$$

where $Y$ is the random variable with distribution $\left\{b_{j}\right\}_{j \geq 0}$ as defined in Theorem 1.2. The distribution of $\tilde{A}$ is determined by its Laplace functional $\varphi(s)$ in (3.10) below.

(b) Moreover, let $r_{t, i}$ be the remaining lifetime of the ith individual alive at time $t$. Let

$$
R(t) \equiv\left\{r_{t, i}: 1 \leq i \leq Z(t)\right\} .
$$

Then, conditioned on the event $\{Z(t)>0\}$, the point process $R(t)$ converges in distribution as $t \rightarrow \infty$ to a point process

$$
\tilde{R} \equiv\left\{\tilde{r}_{i}: 1 \leq i \leq Y\right\},
$$

where $Y$ is as in (a) above. The distribution of $\tilde{R}$ is determined by its Laplace functional in (3.2) below. 
The above theorem can be used to prove Theorem 2.2 below on the coalescence problem for a subcritical Bellman-Harris branching process.

Theorem 2.2. Let $0<m<1$ and $\sum_{j=1}^{\infty}(j \log j) p_{j}<\infty$. Assume that the lifetime distribution $G$ is nonlattice, $G(0+)=0$, the Malthusian parameter $\alpha$ exists, and $\int_{0}^{\infty} t \mathrm{e}^{-\alpha t} \mathrm{~d} G(t)<\infty$. Let $D_{k}(t)$ be as defined in Section 1.3. Then, conditioned on $\{Z(t) \geq 2\}$,

$$
t-D_{2}(t) \stackrel{\mathrm{D}}{\rightarrow} \tilde{D}_{2} \text { as } t \rightarrow \infty
$$

where $\tilde{D}_{2}$ is a positive random variable such that $\mathbb{P}\left(0<\tilde{D}_{2}<\infty\right)=1$. For any $u \geq 0$,

$$
\mathbb{P}\left(\tilde{D}_{2} \leq u\right)=1-\frac{1}{\mathrm{e}^{\alpha u} \mathbb{P}(Y \geq 2)} \mathbb{E}(\phi(\tilde{A}, u)) \equiv H_{2}(u),
$$

where $\tilde{A}$ and $Y$ are as defined in Theorem 2.1,

$$
\phi\left(\left(a_{1}, a_{2}, \ldots, a_{k}\right), u\right)=\mathbb{E}\left(\frac{\sum_{i \neq j=1}^{k} \tilde{Z}_{i}\left(a_{i}+u\right) \tilde{Z}_{j}\left(a_{j}+u\right)}{\left(\sum_{i=1}^{k} \tilde{Z}_{i}\left(a_{i}+u\right)\right)\left(\sum_{i=1}^{k} \tilde{Z}_{i}\left(a_{i}+u\right)-1\right)} \mathbf{1}_{\left\{\sum_{i=1}^{k} \tilde{Z}_{i}\left(a_{i}+u\right) \geq 2\right\}}\right)
$$

for any positive integer $k$ and any positive real numbers $a_{1}, a_{2}, \ldots, a_{k}$, and $\left\{\tilde{Z}_{i}(t): t \geq 0\right\}_{i \geq 1}$ are i.i.d. copies of $\{Z(t): t \geq 0\}$ with newborn initial ancestors.

Remark 2.1. Coalescence thus takes place close to the present.

By the same lines as the proof of Theorem 2.2, we can extend the result to any integer $k \geq 2$.

Corollary 2.1. Let $0<m<1$ and $\sum_{j=1}^{\infty}(j \log j) p_{j}<\infty$. Then, under the same hypotheses as in Theorem 2.2, for any $k \geq 2, t-D_{k}(t) \stackrel{\mathrm{D}}{\rightarrow} \tilde{D}_{k}$ as $t \rightarrow \infty$, where $\tilde{D}_{k}$ is a positive random variable such that $\mathbb{P}\left(0<\tilde{D}_{k}<\infty\right)=1$.

\section{Proofs of the main results}

\subsection{Proof of Theorem 2.1(a)}

Let $Z(t)$ be a continuous-time, single-type, age-dependent Bellman-Harris branching process with $Z(0)=1$. Let $a_{t, i}$ be the age of the $i$ th individual alive at time $t, i=1,2, \ldots, Z(t)$.

To establish Theorem 2.1, it suffices (see Theorem 4.2 of [6]) to show that, for any bounded and continuous function $h: \mathbb{R}_{+} \rightarrow \mathbb{R}_{+}$and $s \geq 0$,

$$
\varphi(s) \equiv \lim _{t \rightarrow \infty} \mathbb{E}\left(\exp \left[-s \sum_{i=1}^{Z(t)} h\left(a_{t, i}\right)\right] \mid Z(t)>0\right)
$$

exists and $\varphi(0+)=1$.

$$
\begin{aligned}
\text { Now, } 0 & \leq s<\infty, t>0 \\
\tilde{H}(s, t) & \equiv \mathbb{E}\left(\exp \left[-s \sum_{i=1}^{Z(t)} h\left(a_{t, i}\right)\right] \mid Z(t)>0\right) \\
& =\frac{1}{\mathbb{P}(Z(t)>0)} \mathbb{E}\left(\exp \left[-s \sum_{i=1}^{Z(t)} h\left(a_{t, i}\right)\right] \mathbf{1}_{\{Z(t)>0\}}\right) \\
& =\frac{1}{\mathbb{P}(Z(t)>0)}\left[\mathbb{E}\left(\exp \left[-s \sum_{i=1}^{Z(t)} h\left(a_{t, i}\right)\right]\right)-\mathbb{E}\left(\exp \left[-s \sum_{i=1}^{Z(t)} h\left(a_{t, i}\right)\right] \mathbf{1}_{\{Z(t)=0\}}\right)\right]
\end{aligned}
$$




$$
\begin{aligned}
& =\frac{1}{\mathbb{P}(Z(t)>0)}\left[\mathbb{E}\left(\exp \left[-s \sum_{i=1}^{Z(t)} h\left(a_{t, i}\right)\right]\right)-\mathbb{E}\left(\mathbf{1}_{\{Z(t)=0\}}\right)\right] \\
& =\frac{1}{\mathbb{P}(Z(t)>0)}\left[\mathbb{E}\left(\exp \left[-s \sum_{i=1}^{Z(t)} h\left(a_{t, i}\right)\right]\right)-1+1-\mathbb{P}(Z(t)=0)\right] \\
& =\frac{1}{\mathbb{P}(Z(t)>0)}\left[\mathbb{E}\left(\exp \left[-s \sum_{i=1}^{Z(t)} h\left(a_{t, i}\right)\right]\right)-1+\mathbb{P}(Z(t)>0)\right] \\
& =\frac{1}{\mathbb{P}(Z(t)>0)}\left[\mathbb{E}\left(\exp \left[-s \sum_{i=1}^{Z(t)} h\left(a_{t, i}\right)\right]\right)-1\right]+1 .
\end{aligned}
$$

Let the ancestor be newborn. Then

$$
\begin{aligned}
H(s, t) & \equiv \mathbb{E}\left(\exp \left[-s \sum_{i=1}^{Z(t)} h\left(a_{t, i}\right)\right]\right) \\
& =\mathbb{E}\left(\exp \left[-s \sum_{i=1}^{Z(t)} h\left(a_{t, i}\right)\right]: L_{0}>t\right)+\mathbb{E}\left(\exp \left[-s \sum_{i=1}^{Z(t)} h\left(a_{t, i}\right)\right]: L_{o} \leq t\right) \\
& =\mathrm{e}^{-\operatorname{sh}(t)} \mathbb{P}\left(L_{0}>t\right)+\int_{[0, t]} f(H(s, t-u)) \mathrm{d} G(u) \\
& =\mathrm{e}^{-\operatorname{sh}(t)}(1-G(t))+\int_{[0, t]} f(H(s, t-u)) \mathrm{d} G(u),
\end{aligned}
$$

which implies that, for any $s \geq 0, H(s, t)$ satisfies the integral equation

$$
H(s, t)=\mathrm{e}^{-s h(t)}(1-G(t))+\int_{[0, t]} f(H(s, t-u)) \mathrm{d} G(u), \quad H(s, 0)=\mathrm{e}^{-s h(0)} .
$$

Moreover,

$$
H(\infty, t) \equiv \lim _{s \rightarrow \infty} H(s, t)=\mathbb{P}(Z(t)=0)
$$

Then, by (3.1) and (3.2),

$$
\begin{aligned}
& \mathbb{E}\left(\exp \left[-s \sum_{i=1}^{Z(t)} h\left(a_{t, i}\right)\right] \mid Z(t)>0\right) \\
& \quad=1-\frac{1}{\mathbb{P}(Z(t)>0)}[1-H(s, t)] \\
& \quad=1-\frac{1}{\mathbb{P}(Z(t)>0)}\left[1-\mathrm{e}^{-s h(t)}(1-G(t))-\int_{[0, t]} f(H(s, t-u)) \mathrm{d} G(u)\right] \\
& \quad=1-\frac{1}{\mathbb{P}(Z(t)>0)}\left[\left(1-\mathrm{e}^{-\operatorname{sh}(t)}\right)(1-G(t))+\int_{[0, t]}[1-f(H(s, t-u))] \mathrm{d} G(u)\right]
\end{aligned}
$$


For any fixed $s \geq 0$, let

$$
\begin{aligned}
& H(t) \equiv 1-H(s, t), \\
& \xi_{1}(t) \equiv\left(1-\mathrm{e}^{-s h(t)}\right)(1-G(t)), \\
& \xi_{2}(t) \equiv \int_{0}^{t}[1-f(H(s, t-u))-m H(t-u)] \mathrm{d} G(u), \\
& \xi_{3}(t) \equiv \xi_{1}(t)+\xi_{2}(t) .
\end{aligned}
$$

Then

$$
H(t)=\xi_{3}(t)+m \int_{[0, t]} H(t-u) \mathrm{d} G(u) .
$$

Before we proceed to the proof of the theorem, we require the introduction of direct Riemann integrability and some lemmas about the properties of the functions defined above.

Definition 3.1. A function $\xi$ is directly Riemann integrable if

(a) $\sum_{n=0}^{\infty} \delta\left(\sup _{n \delta \leq t<(n+1) \delta} \xi(t)\right)$ and $\sum_{n=0}^{\infty} \delta\left(\inf _{n \delta \leq t<(n+1) \delta} \xi(t)\right)$ converge absolutely for sufficient small $\delta>0$; and

(b) $\left(\sum_{n=0}^{\infty} \delta\left(\sup _{n \delta \leq t<(n+1) \delta} \xi(t)\right)-\sum_{n=0}^{\infty} \delta\left(\inf _{n \delta \leq t<(n+1) \delta} \xi(t)\right)\right) \rightarrow 0$ as $\delta \rightarrow 0$.

Remark 3.1. Some sufficient conditions for the direct Riemann integrability of $\xi$ are

(a) $\xi \geq 0$, bounded, continuous, and $\sum_{n=0}^{\infty}\left(\sup _{n \leq t<n+1} \xi(t)\right)<\infty$;

(b) $\xi \geq 0$, nonincreasing, and Riemann integrable in the ordinary sense;

(c) $\xi$ is bounded by a directly Riemann integrable function;

(d) $\xi$ is constant on the intervals $(n, n+1)$ and absolutely integrable.

Lemma 3.1 is a well-known result in renewal theory. See [4, pp. 362-363].

Lemma 3.1. Let $G$ be a probability distribution function, and let $G^{* n}$ denote its $n$-fold convolution. Let $U=\sum_{n=0}^{\infty} G^{* n}$. If $\xi$ is directly Riemann integrable and $G$ is nonlattice, then

$$
\lim _{t \rightarrow \infty}(\xi * U)(t)=\frac{\int_{0}^{\infty} \xi(u) \mathrm{d} u}{\int_{0}^{\infty} u \mathrm{~d} G(u)} .
$$

Lemma 3.2. If the Mathusian parameter $\alpha$ of $m$ and $G$ exists, if $\mathrm{e}^{-\alpha t} \xi(t)$ is directly Riemann integrable, and if $G$ is nonlattice, then the solution $H$ of the integral equation

$$
H(t)=\xi(t)+m \int_{0}^{t} H(t-u) \mathrm{d} G(u), \quad t \geq 0,
$$

satisfies

$$
H(t) \mathrm{e}^{-\alpha t} \rightarrow \frac{\int_{0}^{\infty} \mathrm{e}^{-\alpha u} \xi(t) \mathrm{d} u}{m \int_{0}^{\infty} u \mathrm{e}^{-\alpha u} \mathrm{~d} G(u)}
$$

as $t \rightarrow \infty$.

The proof of Lemma 3.2 can be found in [3]. 
Lemma 3.3. Let $H$ be the function defined in (3.3). Then, under the hypotheses of Theorem 2.1,

$$
\sup _{s, t \geq 0} \mathrm{e}^{-\alpha t} H(t)<\infty .
$$

Proof. For any fixed $s \geq 0$ and any $t \geq 0$, we have

$$
\begin{aligned}
|H(t)| & =|1-H(s, t)| \\
& =\left|\left(1-\mathrm{e}^{-s h(t)}\right)(1-G(t))+\int_{[0, t]}[1-f(H(s, t-u))] \mathrm{d} G(u)\right| \\
& \leq\left|1-\mathrm{e}^{-s h(t)}\right||1-G(t)|+\left|\int_{[0, t]}[1-f(H(s, t-u))] \mathrm{d} G(u)\right| .
\end{aligned}
$$

Note that $f(1)=1,0<H(s, t-u)<1$, and $f$ is a continuous function. Then, by the mean value theorem, there exists $c$ such that $H(s, t-u)<c<1$ and

$$
f^{\prime}(c)=\frac{f(1)-f(H(s, t-u))}{1-H(s, t-u)} .
$$

Therefore,

$$
\begin{aligned}
|H(t)| & \leq\left|1-\mathrm{e}^{-s h(t)}\right||1-G(t)|+\left|\int_{[0, t]} f^{\prime}(c)(1-H(s, t-u)) \mathrm{d} G(u)\right| \\
& \leq|1-G(t)|+\int_{[0, t]}\left|f^{\prime}(c)\right||1-H(s, t-u)| \mathrm{d} G(u) \\
& \leq(1-G(t))+m \int_{[0, t]}|H(t-u)| \mathrm{d} G(u)
\end{aligned}
$$

since $f^{\prime}$ is nondecreasing.

Let $m \mathrm{e}^{-\alpha t} \mathrm{~d} G(t)=\mathrm{d} G_{\alpha}(t)$ and $g_{\alpha}(t) \equiv \mathrm{e}^{-\alpha t}(1-G(t))$. Note that $\int_{0}^{\infty} t \mathrm{e}^{-\alpha t} \mathrm{~d} G(t)<\infty$ implies the Riemann integrability of $g_{\alpha}$. So, $g_{\alpha} \geq 0$ is nonincreasing and Riemann integrable, and, hence, $g_{\alpha}$ is directly Riemann integrable by Remark 3.1(b). Moreover, that $G$ is nonlattice implies that $G_{\alpha}$ is also nonlattice.

Let $G_{\alpha}^{* n}$ be the $n$-fold convolution of $G_{\alpha}$, and let $U_{\alpha}=\sum_{n=0}^{\infty} G_{\alpha}^{* n}$. Then, by Lemma 3.1, we have

$$
\lim _{t \rightarrow \infty} g_{\alpha} * U_{\alpha}(t)=\frac{\int_{0}^{\infty} g_{\alpha}(u) \mathrm{d} u}{\int_{0}^{\infty} u \mathrm{~d} G_{\alpha}(u)}<\infty .
$$

Multiply both sides of (3.7) by $\mathrm{e}^{-\alpha t}$. Then

$$
\begin{aligned}
\mathrm{e}^{-\alpha t}|H(t)| & \leq \mathrm{e}^{-\alpha t}(1-G(t))+m \int_{[0, t]} \mathrm{e}^{-\alpha t}|H(t-u)| \mathrm{d} G(u) \\
& =g_{\alpha}(t)+\int_{[0, t]} \mathrm{e}^{-\alpha(t-u)}|H(t-u)| \mathrm{d} G_{\alpha}(u) \\
& =g_{\alpha}(t)+H_{\alpha} * G_{\alpha}(t) \\
& \leq g_{\alpha}(t)+\left(g_{\alpha}+H_{\alpha} * G_{\alpha}\right) * G_{\alpha}(t) \\
& =\cdots \\
& =g_{\alpha}(t)+g_{\alpha} * G_{\alpha}(t)+g_{\alpha} * G_{\alpha}^{* 2}(t)+g_{\alpha} * G_{\alpha}^{* 3}(t)+\cdots \\
& =g_{\alpha} * U_{\alpha}(t)
\end{aligned}
$$


and, hence, $\lim _{t \rightarrow \infty} \mathrm{e}^{-\alpha t}|H(t)|$ is bounded by a constant for any $s \geq 0$. So,

$$
\sup _{s, t \geq 0} \mathrm{e}^{-\alpha t} H(t)<\infty .
$$

Lemma 3.4. Let $\xi_{1}$ be the function defined in (3.4). Then, under the hypotheses of Theorem 2.1, $\mathrm{e}^{-\alpha t} \xi_{1}(t)$ is directly Riemann integrable.

Proof. Note that

$$
\left|\mathrm{e}^{-\alpha t} \xi_{1}\right|=\left|\mathrm{e}^{-\alpha t}\left(1-\mathrm{e}^{-s h(t)}\right)(1-G(t))\right| \leq \mathrm{e}^{-\alpha t}(1-G(t)) \equiv g_{\alpha}(t),
$$

where $g_{\alpha}$ is known as a directly Riemann integrable function from the proof of Lemma 3.3.

So, $\mathrm{e}^{-\alpha t} \xi_{1}$ is directly Riemann integrable by Remark 3.1(c).

Lemma 3.5. Let $\xi_{2}$ be the function defined in (3.5). Then, under the hypotheses of Theorem 2.1,

$$
\int_{0}^{\infty} \mathrm{e}^{-\alpha t}\left|\xi_{2}(t)\right| \mathrm{d} t<\infty
$$

Proof. Recall that

$$
H(t)=\xi_{1}(t)+\xi_{2}(t)+m \int_{[0, t]} H(t-u) \mathrm{d} G(u)
$$

implies that

$$
\mathrm{e}^{-\alpha t} H(t)=\mathrm{e}^{-\alpha t} \xi_{1}(t)+\mathrm{e}^{-\alpha t} \xi_{2}(t)+m \int_{[0, t]} \mathrm{e}^{-\alpha t} H(t-u) \mathrm{d} G(u) .
$$

Let $H_{\alpha}(t)=\mathrm{e}^{-\alpha t} H(t), \xi_{1 \alpha}(t)=\mathrm{e}^{-\alpha t} \xi_{1}(t)$, and $\xi_{2 \alpha}(t)=\mathrm{e}^{-\alpha t} \xi_{2}(t)$. Then

$$
\begin{aligned}
H_{\alpha}(t) & =\xi_{1 \alpha}(t)+\xi_{2 \alpha}(t)+\int_{[0, t]} H_{\alpha}(t-u) \mathrm{d} G_{\alpha}(u) \\
& =\xi_{1 \alpha}(t)+\xi_{2 \alpha}(t)+H_{\alpha} * G_{\alpha}(t) .
\end{aligned}
$$

We know that $\xi_{1 \alpha}$ is bounded by 1 and, by Lemma $3.3, H_{\alpha}$ is also bounded, so $\xi_{2, \alpha}$ is bounded. Taking Laplace transforms on both sides of (3.8) yields

$$
\hat{H}_{\alpha}(\theta)=\hat{\xi}_{1 \alpha}(\theta)+\hat{\xi}_{2 \alpha}(\theta)+\hat{H}_{\alpha} \cdot \hat{G}_{\alpha}(\theta)
$$

which implies that

$$
\tilde{H}_{\alpha}(\theta)\left(1-\hat{G}_{\alpha}(\theta)\right)+\left(-\tilde{\xi}_{2 \alpha}(\theta)\right)=\hat{\xi}_{1 \alpha}(\theta)
$$

Note that

$$
\frac{f(1)-f(H(s, t-u))}{1-H(s, t-u)}=f^{\prime}(c)<f^{\prime}(1)=m,
$$

and, hence, $\xi_{2}(t)=\int_{0}^{t}[1-f(H(s, t-u))-m H(t-u)] \mathrm{d} G(u)<0$.

So, we have $H_{\alpha} \geq 0, \xi_{1 \alpha} \geq 0, \xi_{2 \alpha} \leq 0$, and $G_{\alpha} \leq 1$. Thus, $\hat{H}_{\alpha}(\theta)\left(1-\hat{G}_{\alpha}(\theta)\right) \geq 0$, $-\hat{\xi}_{2 \alpha}(\theta) \geq 0$, and $\hat{\xi}_{1 \alpha}(\theta) \geq 0$.

Moreover, by the monotone convergence theorem,

$$
\lim _{\theta \downarrow 0} \hat{\xi}_{1 \alpha}(\theta)=\lim _{\theta \downarrow 0} \int_{0}^{\infty} \mathrm{e}^{-\theta t} \xi_{1 \alpha}(t) \mathrm{d} t=\int_{0}^{\infty} \xi_{1 \alpha}(t) \mathrm{d} t=\int_{0}^{\infty} \mathrm{e}^{-\alpha t} \xi_{1}(t) \mathrm{d} t<\infty,
$$


and, hence, $\lim _{\theta \downarrow 0}\left(-\hat{\xi}_{2 \alpha}(\theta)\right)<\infty$ since $\hat{H}_{\alpha}(\theta)\left(1-\hat{G}_{\alpha}(\theta)\right),-\hat{\xi}_{2 \alpha}(\theta)$, and $\hat{\xi}_{1 \alpha}(\theta)$ are of the same sign. Therefore, by the monotone convergence theorem again,

$$
\begin{aligned}
\int_{0}^{\infty} \mathrm{e}^{-\alpha t}\left|\xi_{2}(t)\right| \mathrm{d} t & =\int_{0}^{\infty} \mathrm{e}^{-\alpha t}\left(-\xi_{2}(t)\right) \mathrm{d} t \\
& =\int_{0}^{\infty}\left(-\xi_{2 \alpha}(t)\right) \mathrm{d} t \\
& =\lim _{\theta \downarrow 0} \int_{0}^{\infty} \mathrm{e}^{-\theta t}\left(-\xi_{2 \alpha}(t)\right) \mathrm{d} t \\
& =\lim _{\theta \downarrow 0}\left(-\hat{\xi}_{2 \alpha}(\theta)\right) \\
& <\infty .
\end{aligned}
$$

Lemma 3.6. Let $\xi_{2}$ be the function defined in (3.5). Then, under the hypotheses of Theorem 2.1, $\mathrm{e}^{-\alpha t} \xi_{2}(t)$ is directly Riemann integrable.

Proof. Note that $\alpha<0$. For $n \leq t<n+1$, we have

$$
\begin{aligned}
\mathrm{e}^{-\alpha t}\left|\xi_{2}(t)\right| \leq & \mathrm{e}^{-\alpha(n+1)}\left|\int_{0}^{t}[1-f(H(s, t-u))-m H(t-u)] \mathrm{d} G(u)\right| \\
= & \mathrm{e}^{-\alpha(n+1)} \mid \int_{0}^{n}[1-f(H(s, t-u))-m H(t-u)] \mathrm{d} G(u) \\
& \quad+\int_{n}^{t}[1-f(H(s, t-u))-m H(t-u)] \mathrm{d} G(u) \mid \\
\leq & \mathrm{e}^{-\alpha(n+1)}\left|\int_{0}^{n}[1-f(H(s, n-u))-m H(n-u)] \mathrm{d} G(u)\right| \\
& \quad+\mathrm{e}^{-\alpha(n+1)} \int_{n}^{t}|1-f(H(s, t-u))-m H(t-u)| \mathrm{d} G(u) \\
\leq & \mathrm{e}^{-\alpha} \mathrm{e}^{-n}\left|\xi_{2}(n)\right|+\mathrm{e}^{-\alpha} \mathrm{e}^{-n}(G(1)-G(n))
\end{aligned}
$$

since $0 \leq f \leq 1,0 \leq H \leq 1$, and $0<m<1$. Then

$$
|1-f(H(s, t-u))-m H(t-u)| \leq 1 .
$$

Moreover, by Lemma 3.4, we know that $\int_{0}^{\infty} \mathrm{e}^{-\alpha t}\left|\xi_{2}(t)\right| \mathrm{d} t<\infty$ and, by the assumption, we also have $\int_{0}^{\infty} \mathrm{e}^{-\alpha t}(1-G(t)) \mathrm{d} t<\infty$. So,

$$
\sum_{n=0}^{\infty} \mathrm{e}^{-\alpha n}\left|\xi_{2}(n)\right|<\infty \text { and } \sum_{n=0}^{\infty} \mathrm{e}^{-\alpha n}(1-G(n))<\infty
$$

and, hence,

$$
\sum_{n=0}^{\infty} \sup _{n \leq t<n+1} \mathrm{e}^{-\alpha t}\left|\xi_{2}(t)\right|<\infty .
$$

Since $\mathrm{e}^{-\alpha t}\left|\xi_{2}(t)\right|$ is continuous and bounded, by Remark 3.1(a), $\mathrm{e}^{-\alpha t}\left|\xi_{2}(t)\right|$ is directly Riemann integrable. Therefore, by Remark 3.1(c), $\mathrm{e}^{-\alpha t} \xi_{2}(t)$ is also directly Riemann integrable. 
Now, we are ready to complete the rest of the proof.

By Lemma 3.4 and Lemma 3.6,

$$
\mathrm{e}^{-\alpha t} \xi_{3}(t)=\mathrm{e}^{-\alpha t} \xi_{1}(t)+\mathrm{e}^{-\alpha t} \xi_{2}(t) \text { is directly Riemann integrable. }
$$

Then, by Lemma 3.2, we know that the solution $H$ of the integral equation

$$
H(t)=\xi_{3}(t)+m \int_{[0, t]} H(t-u) \mathrm{d} G(u)
$$

satisfies

$$
H(t) \sim c(s) \mathrm{e}^{\alpha t} \quad \text { as } t \rightarrow \infty
$$

where

$$
c(s)=\frac{\int_{0}^{\infty} \mathrm{e}^{-\alpha u} \xi_{3}(u) \mathrm{d} u}{m \int_{0}^{\infty} u \mathrm{e}^{-\alpha u} \mathrm{~d} G(u)} .
$$

Recall that $\xi_{3}(u)$ is a function of both $s$ and $u$ defined in (3.6). Then

$$
\begin{aligned}
\lim _{t \rightarrow \infty} \mathbb{E}\left(\exp \left[-s \sum_{i=1}^{Z(t)} h\left(a_{t, i}\right)\right] \mid Z(t)>0\right) & =\lim _{t \rightarrow \infty}\left(1-\frac{1}{\mathbb{P}(Z(t)>0)}(1-H(s, t))\right) \\
& =1-\lim _{t \rightarrow \infty} \frac{1}{\mathrm{e}^{-\alpha t} \mathbb{P}(Z(t)>0)}(1-H(s, t)) \mathrm{e}^{-\alpha t} \\
& =1-\lim _{t \rightarrow \infty} \frac{1}{\mathrm{e}^{-\alpha t} \mathbb{P}(Z(t)>0)} H(t) \mathrm{e}^{-\alpha t} \\
& =1-\frac{c(s)}{Q(0)} \\
& \equiv \varphi(s)
\end{aligned}
$$

where $Q$ is as defined in Theorem 1.1 and $c(s)$ is as defined in (3.9).

Moreover, since, by the bounded convergence theorem,

$$
\lim _{s \rightarrow 0+} H(s, t)=\lim _{s \rightarrow 0+} \mathbb{E}\left(\exp \left[-s \sum_{i=1}^{Z(t)} h\left(a_{t, i}\right)\right]\right)=1,
$$

we have

$$
\lim _{s \rightarrow 0+} H(t)=\lim _{s \rightarrow 0+} 1-H(s, t)=0
$$

and

$$
\lim _{s \rightarrow 0+} \xi_{1}(t)=\lim _{s \rightarrow 0+}\left(1-\mathrm{e}^{-s h(t)}\right)(1-G(t))=0 .
$$

Again, by the bounded convergence theorem,

$$
\lim _{s \rightarrow 0+} \xi_{2}(t)=\lim _{s \rightarrow 0+} \int_{0}^{t}(1-f(H(s, t-u)))-m H(t-u) \mathrm{d} G(u)=0 .
$$

Hence, $\lim _{s \rightarrow 0+} \xi_{3}(t)=\lim _{s \rightarrow 0+}\left(\xi_{1}(t)+\xi_{2}(t)\right)=0$.

Also, for any $s \geq 0,\left|\mathrm{e}^{-\alpha t} \xi_{3}(t)\right| \leq \mathrm{e}^{-\alpha t}\left|\xi_{1}(t)\right|+\mathrm{e}^{-\alpha t}\left|\xi_{2}(t)\right|$, where $\mathrm{e}^{-\alpha t}\left|\xi_{1}(t)\right|$ and $\mathrm{e}^{-\alpha t}\left|\xi_{2}(t)\right|$ are integrable. Then, by the dominated convergence theorem,

$$
\lim _{s \rightarrow 0+} \int_{0}^{\infty} \mathrm{e}^{-\alpha t} \xi_{3}(t) \mathrm{d} t=\int_{0}^{\infty} \lim _{s \rightarrow 0+} \mathrm{e}^{-\alpha t} \xi_{3}(t) \mathrm{d} t=0,
$$


and, hence,

$$
\lim _{s \rightarrow 0+} \varphi(s)=\lim _{s \rightarrow 0+} 1-\frac{c(s)}{Q(0)}=1-\lim _{s \rightarrow 0+} \frac{1}{Q(0)} \frac{\int_{0}^{\infty} \mathrm{e}^{-\alpha u} \xi_{3}(u) \mathrm{d} u}{m \int_{0}^{\infty} u \mathrm{e}^{-\alpha u} \mathrm{~d} G(u)}=1-0=1 .
$$

Therefore, $\varphi$ is a Laplace functional of a point process (see [4, pp. 429-434]).

Since, for any $s \geq 0$,

$$
\varphi(s)=\lim _{t \rightarrow \infty} \mathbb{E}\left(\exp \left[-s \sum_{i=1}^{Z(t)} h\left(a_{t, i}\right)\right] \mid Z(t)>0\right)
$$

and, by Theorem 1.2,

$$
Z(t) \mid Z(t)>0 \stackrel{\mathrm{D}}{\rightarrow} Y \quad \text { as } t \rightarrow \infty,
$$

there exists a point process $\tilde{A} \equiv\left\{\tilde{a}_{i}: 1 \leq i \leq Y\right\}$ such that

$$
\varphi(s)=\mathbb{E}\left(\exp \left[-s \sum_{i=1}^{Y} h\left(\tilde{a}_{i}\right)\right]\right)
$$

for any $s \geq 0$, and, as $t \rightarrow \infty$,

$$
A(t) \mid Z(t)>0 \stackrel{\mathrm{D}}{\rightarrow} \tilde{A}
$$

This completes the proof of Theorem 2.1(a).

Remark 3.2. A more detailed study of $\tilde{A}$ is an interesting open problem.

\subsection{Proof of Theorem 2.1(b)}

Let $h:[0, \infty) \rightarrow[0, \infty)$ be a continuous function. Let

$$
\tilde{K}(s, t) \equiv \mathbb{E}\left(\exp \left[-s \sum_{i=1}^{Z(t)} h\left(r_{t, i}\right)\right] \mid Z(t)>0\right) .
$$

Then

$$
\tilde{K}(s, t)=\mathbb{E}\left(\mathbb{E}\left(\exp \left[-s \sum_{i=1}^{Z(t)} h\left(r_{t, i}\right)\right] \mid A(t), Z(t)>0\right) \mid Z(t)>0\right) .
$$

Now, for $t>0$,

$$
\mathbb{E}\left(\exp \left[-s \sum_{i=1}^{Z(t)} h\left(r_{t, i}\right)\right] \mid A(t)\right)=\prod_{i=1}^{Z(t)} \psi\left(s, a_{t, i}\right)
$$

where

$$
\psi(s, x) \equiv \mathbb{E}\left(\mathrm{e}^{-s h\left(r_{t, i}\right)} \mid a_{t, i}=x\right)=\int_{[0, \infty)} \mathrm{e}^{-\operatorname{sh}(y)} \frac{\mathrm{d} G(x+y)}{1-G(x)}
$$

for $0<x<\infty$ and $0 \leq s<\infty$. So,

$$
\mathbb{E}\left(\exp \left[-s \sum_{i=1}^{Z(t)} h\left(r_{t, i}\right)\right] \mid A(t)\right)=\exp \left[-\sum_{i=1}^{Z(t)}\left(-\log \psi\left(s, a_{t, i}\right)\right)\right]
$$


Since $-\log \psi(s, x)$ is a positive continuous function of $x$, by Theorem 2.1(a), we have, as $t \rightarrow \infty$

$$
\begin{aligned}
\tilde{K}(s, t) & =\mathbb{E}\left(\exp \left[-\sum_{i=1}^{Z(t)}\left(-\log \psi\left(s, a_{t, i}\right)\right)\right] \mid Z(t)>0\right) \\
& \rightarrow \mathbb{E}\left(\exp \left[-\sum_{i=1}^{Y}\left(-\log \psi\left(s, \tilde{a}_{i}\right)\right)\right]\right) .
\end{aligned}
$$

Also, by the bounded convergence theorem,

$$
\lim _{s \rightarrow 0+} \mathbb{E}\left(\exp \left[-\sum_{i=1}^{Y}\left(-\log \psi\left(s, \tilde{a}_{i}\right)\right)\right]\right)=1,
$$

and, hence,

$$
\mathbb{E}\left(\exp \left[-\sum_{i=1}^{Y}\left(-\log \psi\left(s, \tilde{a}_{i}\right)\right)\right]\right)
$$

is a Laplace functional of a point process. Therefore, there exists a point process $\tilde{R} \equiv\left\{\tilde{r}_{i}: 1 \leq\right.$ $i \leq Y\}$ such that

$$
\mathbb{E}\left(\exp \left[-s \sum_{i=1}^{Y} h\left(\tilde{r}_{i}\right)\right]\right)=\lim _{t \rightarrow \infty} \mathbb{E}\left(\exp \left[-s \sum_{i=1}^{Z(t)} h\left(r_{t, i}\right)\right] \mid Z(t)>0\right)
$$

for any $s \geq 0$. That is, as $t \rightarrow \infty$,

$$
R(t) \mid Z(t)>0 \stackrel{\mathrm{D}}{\rightarrow} \tilde{R}
$$

This completes the proof of Theorem 2.1(b).

\subsection{Proof of Theorem 2.2}

Let $\left\{Z_{t, i}(u): u \geq 0\right\}$ be the branching process initiated by the $i$ th individual alive at time $t$. So,

$$
Z(t)=\sum_{i=1}^{Z(t-u)} Z_{t-u, i}\left(a_{t-u, i}+u\right)
$$

For any $u \leq t$,

$$
\begin{aligned}
\mathbb{P}(t- & \left.D_{2}(t) \geq u \mid Z(t) \geq 2\right) \\
= & \mathbb{P}\left(D_{2}(t) \leq t-u \mid Z(t) \geq 2\right) \\
= & \mathbb{E}\left(\frac{\sum_{i \neq j=1}^{Z(t-u)} Z_{t-u, i}\left(a_{t-u, i}+u\right) Z_{t-u, j}\left(a_{t-u, j}+u\right)}{Z(t)(Z(t)-1)} \mid Z(t) \geq 2\right) \\
= & \frac{1}{\mathbb{P}(Z(t) \geq 2)} \mathbb{E}\left(\frac{\sum_{i \neq j=1}^{Z(t-u)} Z_{t-u, i}\left(a_{t-u, i}+u\right) Z_{t-u, j}\left(a_{t-u, j}+u\right)}{Z(t)(Z(t)-1)} \mathbf{1}_{\{Z(t) \geq 2\}}\right) \\
= & \frac{1}{\mathbb{P}(Z(t) \geq 2, Z(t)>0)} \\
& \times \mathbb{E}\left(\frac{\sum_{i \neq j=1}^{Z(t-u)} Z_{t-u, i}\left(a_{t-u, i}+u\right) Z_{t-u, j}\left(a_{t-u, j}+u\right)}{Z(t)(Z(t)-1)} \mathbf{1}_{\{Z(t) \geq 2\}} \mathbf{1}_{\{Z(t-u)>0\}}\right) .
\end{aligned}
$$


By (3.11) and the definition of the conditional probability, we have

$$
\begin{aligned}
\mathbb{P}(t- & \left.D_{2}(t) \geq u \mid Z(t) \geq 2\right) \\
= & \frac{\mathbb{P}(Z(t-u)>0)}{\mathbb{P}(Z(t) \geq 2 \mid Z(t)>0) \mathbb{P}(Z(t)>0)} \\
& \times \mathbb{E}\left(\frac{\sum_{i \neq j=1}^{Z(t-u)} Z_{t-u, i}\left(a_{t-u, i}+u\right) Z_{t-u, j}\left(a_{t-u, j}+u\right)}{\left(\sum_{i=1}^{Z(t-u)} Z_{t-u, i}\left(a_{t-u, i}+u\right)\right)\left(\sum_{i=1}^{Z(t-u)} Z_{t-u, i}\left(a_{t-u, i}+u\right)-1\right)}\right. \\
& \left.\quad \times \mathbf{1}_{\left\{\sum_{i=1}^{Z(t-u)} Z_{t-u, i}\left(a_{t-u, i}+u\right) \geq 2\right\}} \mid Z(t-u)>0\right) \\
= & \frac{\mathbb{P}(Z(t-u)>0)}{\mathbb{P}(Z(t) \geq 2 \mid Z(t)>0) \mathbb{P}(Z(t)>0)} \\
& \times \mathbb{E}\left(\frac{\sum_{i \neq j=1}^{Z(t-u)} \tilde{Z}_{i}\left(a_{t-u, i}+u\right) \tilde{Z}_{j}\left(a_{t-u, j}+u\right)}{\left(\sum_{i=1}^{Z(t-u)} \tilde{Z}_{i}\left(a_{t-u, i}+u\right)\right)\left(\sum_{i=1}^{Z(t-u)} \tilde{Z}_{i}\left(a_{t-u, i}+u\right)-1\right)}\right. \\
& \quad \times \mathbf{1}_{\left.\left\{\sum_{i=1}^{Z(t-u)} \tilde{Z}_{i}\left(a_{t-u, i}+u\right) \geq 2\right\} \mid Z(t-u)>0\right)} \\
= & \frac{1}{\mathbb{P}(Z(t) \geq 2 \mid Z(t)>0)} \frac{\mathbb{P}(Z(t-u)>0)}{\mathbb{P}(Z(t)>0)} \\
& \times \mathbb{E}\left(\phi(A(t-u), u) \mid A(t-u)=\left(a_{t-u, 1}, a_{t-u, 2}, \ldots, a_{t-u, Z(t-u)}\right)\right),
\end{aligned}
$$

where $\left\{\tilde{Z}_{i}(t)\right\}_{i \geq 1}$ are i.i.d. copies of $Z(t)$ and

$$
\phi\left(\left(a_{1}, a_{2}, \ldots, a_{k}\right), u\right)=\mathbb{E}\left(\frac{\sum_{i \neq j=1}^{k} \tilde{Z}_{i}\left(a_{i}+u\right) \tilde{Z}_{j}\left(a_{j}+u\right)}{\left(\sum_{i=1}^{k} \tilde{Z}_{i}\left(a_{i}+u\right)\right)\left(\sum_{i=1}^{k} \tilde{Z}_{i}\left(a_{i}+u\right)-1\right)} \mathbf{1}_{\left\{\sum_{i=1}^{k} \tilde{Z}_{i}\left(a_{i}+u\right) \geq 2\right\}}\right)
$$

for any positive integer $k$ and any positive real numbers $a_{1}, a_{2}, \ldots, a_{k}$.

Since, for any fixed $u, \phi(\cdot, u)$ is bounded and continuous and by Theorem 2.1 (see also [6, pp. 14-15]),

$$
\mathbb{E}(\phi(A(t-u), u) \mid Z(t-u)>0) \rightarrow \mathbb{E}(\phi(\tilde{A}, u))
$$

as $t \rightarrow \infty$, where $\tilde{A}$ is as in (2.1).

Moreover, by Theorem 1.2(b), i.e. $\mathbb{P}(Z(t)>0) \sim c(s) \mathrm{e}^{-\alpha t}$, we have

$$
\begin{aligned}
\lim _{t \rightarrow \infty} & \mathbb{P}\left(t-D_{2}(t)>u \mid Z(t) \geq 2\right) \\
& =\lim _{t \rightarrow \infty} \frac{1}{\mathbb{P}(Z(t) \geq 2 \mid Z(t)>0)} \frac{c(s) \mathrm{e}^{\alpha(t-u)}}{c(s) \mathrm{e}^{\alpha t}} \mathbb{E}\left(\phi_{2}(A(t-u), u) \mid Z(t-u)>0\right) \\
& =\frac{1}{\mathbb{P}(Y \geq 2)} \mathrm{e}^{-\alpha u} \mathbb{E}(\phi(\tilde{A}, u)) \\
& \equiv 1-H_{2}(u) .
\end{aligned}
$$

It remains to show that $H_{2}$ is a proper probability distribution, i.e. $H_{2}(u) \rightarrow 1$ as $u \rightarrow \infty$.

It suffices to prove that

$$
\lim _{u \rightarrow \infty} \mathrm{e}^{-\alpha u} \mathbb{E}(\phi(\tilde{A}, u))=0 .
$$


First, we have

$$
\begin{aligned}
\mathbb{E}(\phi(\tilde{A}, u))= & \mathbb{E}\left(\frac{\sum_{i \neq j=1}^{Y} \tilde{Z}_{i}\left(\tilde{a}_{i}+u\right) \tilde{Z}_{j}\left(\tilde{a}_{j}+u\right)}{\left(\sum_{i=1}^{Y} \tilde{Z}_{i}\left(\tilde{a}_{i}+u\right)\right)\left(\sum_{i=1}^{Y} \tilde{Z}_{i}\left(\tilde{a}_{i}+u\right)-1\right)} \mathbf{1}_{\left\{\sum_{i=1}^{Y} \tilde{Z}_{i}\left(\tilde{a}_{i}+u\right) \geq 2\right\}}\right) \\
= & \mathbb{E}\left(\mathbb{E}\left(\frac{\sum_{i \neq j=1}^{Y} \tilde{Z}_{i}\left(\tilde{a}_{i}+u\right) \tilde{Z}_{j}\left(\tilde{a}_{j}+u\right)}{\left(\sum_{i=1}^{Y} \tilde{Z}_{i}\left(\tilde{a}_{i}+u\right)\right)\left(\sum_{i=1}^{Y} \tilde{Z}_{i}\left(\tilde{a}_{i}+u\right)-1\right)} \mathbf{1}_{\left\{\sum_{i=1}^{Y} \tilde{Z}_{i}\left(a_{i}+u\right) \geq 2\right\}} \mid \tilde{A}\right)\right) \\
\leq & \mathbb{E}\left(\mathbb { P } \left(\text { there exist } 1 \leq i, j \leq Y \text { such that } i \neq j, \tilde{Z}_{i}\left(\tilde{a}_{i}+u\right)>0,\right.\right. \\
\leq & \quad \mathbb{E}\left(1-\mathbb{P}\left(\tilde{Z}_{i}\left(\tilde{a}_{i}+u\right)=0 \text { for all } i=1,2, \ldots, Y \mid \tilde{A}\right)\right. \\
& \left.\quad-\mathbb{P}\left(\tilde{Z}_{i}\left(\tilde{a}_{i}+u\right)>0 \text { for some } i \text { and } \tilde{Z}_{j}\left(\tilde{a}_{j}+u\right)=0 \text { for all } j \neq i \mid \tilde{A}\right)\right) .
\end{aligned}
$$

For any $0 \leq s \leq 1$ and $t \geq 0$, let

$$
F(s, t)=\sum_{j=0}^{\infty} \mathbb{P}(Z(t)=j) s^{j},
$$

and, by Theorem 1.1, we have

$$
\lim _{t \rightarrow \infty} \mathrm{e}^{-\alpha t}(1-F(s, t)) \equiv Q(s) \quad \text { exists for } 0 \leq s \leq 1 .
$$

So,

$\mathrm{e}^{-\alpha u} \mathbb{E}(\phi(\tilde{A}, u))$

$$
\leq \mathrm{e}^{-\alpha u} \mathbb{E}\left(1-\prod_{i=1}^{Y} F\left(0, \tilde{a}_{i}+u\right)-\sum_{i=1}^{Y}\left(1-F\left(0, \tilde{a}_{i}+u\right)\right) \prod_{j \neq i} F\left(0, \tilde{a}_{j}+u\right)\right) .
$$

Note that the assumption of $\sum_{j=1}^{\infty}(j \log j) p_{j}<\infty$ implies that $0<\mathbb{E} Y<\infty$ and, hence, $\mathbb{P}(0<Y<\infty)=1$.

Now, conditioned on the limit age chart $\tilde{A}$, we have

$$
\begin{aligned}
\lim _{u \rightarrow \infty} \mathrm{e}^{-\alpha u}\left(1-\prod_{i=1}^{Y} F\left(0, \tilde{a}_{i}+u\right)\right) \\
\quad=\lim _{u \rightarrow \infty} \mathrm{e}^{-\alpha u}\left(1-\prod_{i=1}^{Y}\left(1-Q(0) \mathrm{e}^{\alpha\left(\tilde{a}_{i}+u\right)}\right)\right) \\
=\lim _{u \rightarrow \infty} \frac{1-\prod_{i=1}^{Y}\left(1-Q(0) \mathrm{e}^{\alpha\left(\tilde{a}_{i}+u\right)}\right)}{\mathrm{e}^{\alpha u}} \\
=\lim _{u \rightarrow \infty} \frac{-\sum_{i=1}^{Y}\left(-Q(0) \mathrm{e}^{\alpha \tilde{a}_{i}} \alpha \mathrm{e}^{\alpha u}\right) \prod_{j \neq i}\left(1-Q(0) \mathrm{e}^{\alpha\left(\tilde{a}_{j}+u\right)}\right)}{\alpha \mathrm{e}^{\alpha u}} \\
=\lim _{u \rightarrow \infty} \sum_{i=1}^{Y} Q(0) \mathrm{e}^{\alpha \tilde{a}_{i}} \prod_{j \neq i}\left(1-Q(0) \mathrm{e}^{\alpha\left(\tilde{a}_{j}+u\right)}\right) \\
=Q(0) \sum_{i=1}^{Y} \mathrm{e}^{\alpha \tilde{a}_{i}}
\end{aligned}
$$


and

$$
\begin{aligned}
& \lim _{u \rightarrow \infty} \mathrm{e}^{-\alpha u}\left(\sum_{i=1}^{Y}\left(1-F\left(0, \tilde{a}_{i}+u\right)\right) \prod_{j \neq i} F\left(0, \tilde{a}_{j}+u\right)\right) \\
& =\lim _{u \rightarrow \infty} \mathrm{e}^{-\alpha u}\left(\sum_{i=1}^{Y} Q(0) \mathrm{e}^{\alpha\left(\tilde{a}_{i}+u\right)} \prod_{j \neq i}\left(1-Q(0) \mathrm{e}^{\alpha\left(\tilde{a}_{j}+u\right)}\right)\right) \\
& \geq \lim _{u \rightarrow \infty} \mathrm{e}^{-\alpha u}\left(\sum_{i=1}^{Y} Q(0) \mathrm{e}^{\alpha\left(\tilde{a}_{i}+u\right)} \prod_{j \neq i}\left(1-Q(0) \mathrm{e}^{\alpha u}\right)\right) \\
& =\lim _{u \rightarrow \infty} \mathrm{e}^{-\alpha u}\left(\sum_{i=1}^{Y} Q(0) \mathrm{e}^{\alpha\left(\tilde{a}_{i}+u\right)}\left(1-Q(0) \mathrm{e}^{\alpha u}\right)^{Y-1}\right) \\
& =\lim _{u \rightarrow \infty} \sum_{i=1}^{Y} Q(0) \mathrm{e}^{\alpha \tilde{a}_{i}}\left(1-Q(0) \mathrm{e}^{\alpha u}\right)^{Y-1} \\
& =Q(0) \sum_{i=1}^{Y} \mathrm{e}^{\alpha \tilde{a}_{i}} .
\end{aligned}
$$

Hence, conditioned on $\tilde{A}$,

$$
\begin{aligned}
0 \leq & \lim _{u \rightarrow \infty} \mathrm{e}^{-\alpha u}\left(1-\prod_{i=1}^{Y} F\left(0, \tilde{a}_{i}+u\right)-\sum_{i=1}^{Y}\left(1-F\left(0, \tilde{a}_{i}+u\right)\right) \prod_{j \neq i} F\left(0, \tilde{a}_{j}+u\right)\right) \\
= & \lim _{u \rightarrow \infty} \mathrm{e}^{-\alpha u}\left(1-\prod_{i=1}^{Y} F\left(0, \tilde{a}_{i}+u\right)\right) \\
& -\lim _{u \rightarrow \infty} \mathrm{e}^{-\alpha u}\left(\sum_{i=1}^{Y}\left(1-F\left(0, \tilde{a}_{i}+u\right)\right) \prod_{j \neq i} F\left(0, \tilde{a}_{j}+u\right)\right) \\
\leq & Q(0) \sum_{i=1}^{Y} \mathrm{e}^{\alpha \tilde{a}_{i}}-Q(0) \sum_{i=1}^{Y} \mathrm{e}^{\alpha \tilde{a}_{i}} \\
= & 0 \quad \text { with probability } 1 .
\end{aligned}
$$

Therefore, by the bounded convergence theorem,

$$
\begin{aligned}
\lim _{u \rightarrow \infty} & \mathrm{e}^{-\alpha u} \mathbb{E}(\phi(\tilde{A}, u)) \\
& =\lim _{u \rightarrow \infty} \mathrm{e}^{-\alpha u} \mathbb{E}\left(1-\prod_{i=1}^{Y} F\left(0, \tilde{a}_{i}+u\right)-\sum_{i=1}^{Y}\left(1-F\left(0, \tilde{a}_{i}+u\right)\right) \prod_{j \neq i} F\left(0, \tilde{a}_{j}+u\right)\right) \\
& =0 .
\end{aligned}
$$

This completes the proof.

\section{References}

[1] Athreya, K. B. (2012). Coalescence in the recent past in rapidly growing populations. Stoch. Process. Appl. 122, 3757-3766.

[2] Athreya, K. B. (2012). Coalescence in critical and subcritical Galton-Watson branching processes. J. Appl. Prob. 49, 627-638. 
[3] Athreya, K. B. And Ney, P. E. (2004). Branching Processes. Dover Publications, Mineola, NY.

[4] Feller, W. (1971). An Introduction to Probability Theory and Its Applications, Vol. II, 2nd edn. John Wiley, New York.

[5] Hong, J. (2011). Coalescence in Bellman-Harris and multi-type branching processes. Doctoral Thesis, Iowa State University.

[6] Kallenberg, O. (1986). Random Measures, 4th edn. Akademie, Berlin.

[7] Lambert, A. (2003). Coalescence times for the branching process. Adv. Appl. Prob. 35, 1071-1089. 